\title{
Performance Analysis of Noncoherent Frame Synchronization in Satellite Communications with Frequency Uncertainty
}

\author{
Nicolò Mazzali*, Giulio Stante ${ }^{\dagger}$, Bhavani Shankar Mysore Rama R*, and Björn Ottersten* \\ ${ }^{*}$ Interdisciplinary Centre for Security, Reliability and Trust \\ University of Luxembourg, Luxembourg \\ e-mails: \{nicolo.mazzali, bhavani.shankar, bjorn.ottersten\}@uni.lu \\ ${ }^{\dagger}$ University of Parma, Italy \\ e-mail: giulio.stante@ studenti.unipr.it
}

\begin{abstract}
Emerging markets for satellite communications, such as maritime and aeronautical applications, require reliable communications in challenging conditions and low-cost, lowcomplexity user terminals. In this perspective, the synchronization chain is a fundamental part of the receiver and its design becomes critical. When a quadricorrelator is used as first stage of the synchronization chain so as to perform a coarse frequency estimation, then a noncoherent algorithm for frame synchronization can be employed as second stage. A popular algorithm for frame synchronization is the post-detection integration (PDI), whose noncoherent version (NCPDI) is quite robust to non-negligible residual frequency offsets. In this paper we will assess the performance of NCPDI in presence of a random uniformly-distributed residual frequency offset and will find a closed-form approximation for the probability of detection. Moreover, we will optimize the design parameters of NCPDI for a system operating at very low signal-to-noise ratio (VL-SNR) by minimizing the probability of missed detection for a given probability of false alarm.
\end{abstract}

Index Terms-frame synchronization; noncoherent postdetection integration; random frequency offset

\section{INTRODUCTION}

In the recent years, satellite communications have experienced a remarkable development; new market sectors have triggered research in new scenarios and forced researchers to take into account application-driven constraints, stemming from hardware and commercial perspectives. In particular, maritime and aeronautical applications require highly reliable communications even in extremely challenging conditions, e.g., at very low signal-to-noise ratio (VL-SNR). This is the case, for example, for the forward link of maritime communications in polar regions, where the elevation angle of the satellites is very low. Moreover, such applications also require low-complexity hardware in order to limit the final cost of the equipment needed by the user terminals.

In such scenarios, the design of an efficient synchronization chain is of paramount importance. Indeed, the received signal is impaired by (i) phase noise introduced by the possibly cheap local oscillator of the user terminal, (ii) a frequency offset generated by oscillator misalignment, and (iii) timing errors produced by an inexact sampling. To overcome these impair- ments, many communication standards (e.g., the DVB-S2X [1]) define special frames providing extra pilot fields that can be conveniently exploited for synchronization. Therefore, the correct detection of the start of the superframe, and hence the pilot fields, is necessary for all the following synchronization blocks.

The synchronization problem does not have a unique solution. However, the first block in the synchronization chain usually performs either a coarse frequency estimation or a frame acquisition, leaving timing, fine frequency, and phase estimations for the following blocks. A widely used algorithm for coarse frequency synchronization, completely agnostic of any signal parameter and non pilot-aided, is the quadricorrelator [2]. The output of the coarse frequency estimator has usually a low residual frequency offset (whose value depends on the considered application), and the algorithm for frame acquisition (when performed as second stage in the synchronization chain) has to exhibit a good resilience to small frequency offsets.

Frame acquisition is based on the discretization of the uncertainty region into a finite number of cells, each of which is associated to a hypothesis. In this way, the acquisition problem is transformed into a detection problem where the receiver has to decide between the hypothesis of synchronous cell $\left(H_{1}\right)$ and that of misaligned cell $\left(H_{0}\right)$. In general, the received frame is composed of a known preamble field followed by the payload data field, and frame synchronization is normally performed on the preamble. Such a structure can be applied to both burst and continuous transmissions, while the extra pilot fields (like those provided for example by the DVB-S2X standard [1]) may be exploited to improve its performance. Further, in case of continuous transmission, the frame acquisition can be used in conjunction with a verification stage based on the regularity of the frame structure. However, the verification stage is less critical than the acquisition one [3], and hence it will not be considered in this paper.

Concerning the choice of a suitable algorithm for frame synchronization, post-detection integration (PDI) is one of the most popular solutions [4]. Thorough analyses of PDI and 
its many versions can be found in [3]-[6] and references therein. If the quadricorrelator [2] is used as first stage of the synchronization chain, then the noncoherent version of PDI (NCPDI) can still be used despite the residual frequency offset [6]. Being the simplest among all the PDI versions, NCPDI provides excellent performance with a low computational cost, even when the residual frequency offset is non-negligible [6].

In this paper, we extend the analysis of NCPDI carried out in [7] by assuming a random residual frequency offset. The residual frequency uncertainty after coarse frequency offset correction depends on the system parameters (more so in the considered VL-SNR scenarios), is unknown, and may vary from frame to frame. Optimizing the NCDPI parameters, therefore, calls for taking these variations into account. Towards this, in this paper we model the residual frequency offset as a random variable and derive the average probability of detection. Similar premises were assumed in [6], where the considered test statistic was dependent on the residual frequency offset. By contrast, we will use a test statistic independent of the frequency offset, allowing a significant reduction of the receiver complexity. We will find a closedform approximated expression for the probability of detection, and then optimize the design parameters of the NCPDI in order to maximize the probability of detection for a given probability of false alarm.

\section{SySTEM MOdEL}

Let us consider the received signal on the forward link of a satellite communication system. The system could be broadband or broadcast. Without loss of generality, we assume a generic transmission format with known preamble followed by data. For example, the superframe structure provided by the DVB-S2X standard [1] would fit the considered structure as the start-of-superframe (SoSF) field is located at the beginning of the superframe, as shown in Fig. 1. However, for the sake of generality, we will not exploit the regular repetition of the frame structure (typical of continuous transmissions) but rely on a frame-by-frame detection approach so as to include burst transmissions in the system model. For the sake of analytical

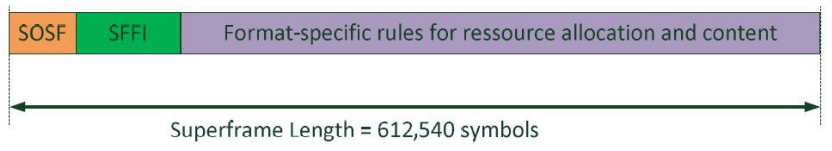

Fig. 1. Superframe structure according to the DVB-S2X standard.

tractability, we will assume the channel to be impaired by additive white Gaussian noise (AWGN) only. In other words, we will neglect the effects, linear and nonlinear, introduced by the satellite transponder, as well as propagation impairments like rain attenuation. Moreover, we will also assume the absence of adjacent channel interference. These assumptions are realistic if the on-board power amplifier (whose characteristics are usually linearized) is sufficiently backed-off from its saturation point, and if the signal bandwidth is smaller than the bandwidths of the on-board filters (input and output multiplexers). This is the case, for example, of a multicarrierper-transponder scenario where the different signals occupy disjoint bands inside the transponder bandwidth.

Under these assumptions, the received signal before the front-end filter is

$$
\begin{aligned}
x_{R}(t) & =s(t)+w(t) \\
& =\alpha \sqrt{E_{s}} \sum_{k=-\infty}^{+\infty} a_{k} p\left(t-k T_{s}\right) e^{\jmath(2 \pi \Delta f t+\varphi)}+w(t)
\end{aligned}
$$

where $E_{s}$ is the average signal energy, $\left\{a_{k}\right\}$ are the transmitted symbols assumed to have mean zero and unit variance (i.e., $E\left\{a_{k}\right\}=0$ and $\left.E\left\{\left|a_{k}\right|^{2}\right\}=1\right), p(t)$ is the shaping pulse, $T_{s}$ the symbol period, $\triangle f$ a random frequency offset uniformly distributed in $\left[-\beta / T_{s}, \beta / T_{s}\right], \varphi$ a random phase offset in $[0,2 \pi)$, and $w(t)$ is the additive white Gaussian noise (AWGN) with power spectral density $2 N_{0}$. In order to model the possible absence of signal, we introduce an auxiliary random variable $\alpha$ distributed as Bernoulli of parameter $p_{s}$, implying that the signal is missing with probability $1-p_{s}$. After a block performing coarse frequency estimation (by means of the quadricorrelator [2]), the normalized maximum residual frequency offset $\beta$ is typically in the range $[0,0.05]$.

As front-end filter, a matched filter is assumed. Therefore, if the frequency offset is small if compared to the signal bandwidth, the filtered signal reads

$$
r(t) \cong \alpha \sqrt{E_{s}} \sum_{k=-\infty}^{+\infty} a_{k} R_{p}\left(t-k T_{s}\right) e^{\jmath(2 \pi \Delta f t+\varphi)}+n(t)
$$

where $R_{p}(t)=p(t) \otimes p^{*}(-t)$ and $n(t)=w(t) \otimes p^{*}(-t)$, and the symbol $\otimes$ denotes convolution. The filtered signal is then sampled at the time instant $\tau=(m+\Delta) T_{s}+\delta$, where $\Delta$ denotes the integer timing offset and $\delta$ the fractional timing offset. In the following, we will assume $\delta \in\left[-T_{s} / 2, T_{s} / 2\right]$ to be unknown but deterministic. Hence, the $m$-th sample reads

$$
\begin{aligned}
r_{m} & =\alpha \sqrt{E_{s}} \sum_{k=-\infty}^{+\infty} a_{k} R_{p}\left[(m+\Delta-k+D) T_{s}\right] \\
& \times e^{\jmath[2 \pi F(m+\Delta+D)+\varphi]}+n_{m}
\end{aligned}
$$

where we have introduced the normalized offsets $F=\Delta f T_{s}$ and $D=\delta / T_{s}$. The noise samples are complex circularlysymmetric Gaussian random variables having $E\left\{n_{m}\right\}=0$, $E\left\{n_{m} n_{k}^{*}\right\}=2 N_{0} R_{p}\left[(m-k) T_{s}\right]$. In the following we assume the shaping pulse $p(t)$ to be a square-root raised cosine (SRRC) pulse. Therefore, $R_{p}\left(n T_{s}\right)=\delta[n]$ where $\delta[n]$ is the Kronecker delta, and the noise correlation becomes $E\left\{n_{m} n_{k}^{*}\right\}=2 N_{0} \delta[n]$. The noise variance will be denoted in the following as $E\left\{\left|n_{m}\right|^{2}\right\}=2 \sigma^{2}$.

\section{Noncoherent Post-Detection Integration (NCPDI) ALGORITHM}

The NCPDI algorithm [4] correlates the received signal with a sequence of known pilot symbols. Let us assume the frame length to be $L_{F}$ symbols, and the length of the pilot sequence to be $L_{P}$ symbols. For the ease of presentation, we assume 
the known symbols to be located at the very beginning of the frame, implying that

$$
a_{k}= \begin{cases}c_{k} & \text { if }|k|_{L_{F}} \in\left[0, L_{P}-1\right] \\ d_{k} & \text { if }|k|_{L_{F}} \in\left[L_{P}, L_{F}-1\right]\end{cases}
$$

where $\left\{c_{k}\right\}$ are the known pilot symbols, $\left\{d_{k}\right\}$ are the unknown information symbols, and $|\cdot|_{N}$ denotes the modulo- $N$ operator. The algorithm computes the correlation

$$
x_{i}=\frac{1}{M} \sum_{m=i M}^{(i+1) M-1} r_{m} c_{m}^{*}
$$

where $m \in\left[0, L_{P}-1\right]$, and $L_{P}=M L$ with $M, L \in \mathbb{N}$. In other words, the portion of the received signal that has to be correlated with the pilot field is partitioned in $L$ sub-blocks formed by $M$ samples each [7]. The correlation $x_{i}$ is computed over each sub-block, then it is scaled as $y_{i}=x_{i} / \sqrt{L}$, and finally accumulated as

$$
\Lambda=\sum_{i=0}^{L-1}\left|y_{i}\right|^{2}
$$

in order to form the test statistic [7]. Finally, the test statistic $\Lambda$ is compared to a threshold $\xi$ so as to decide on signaling the start of a frame [4].

For the hypothesis testing, we define two hypotheses:

- $H_{0}$ : absence of signal (i.e., $\alpha=0$ ) or misalignment (i.e., $\alpha=1$ and $\Delta \neq 0$ );

- $H_{1}$ : correct synchronization (i.e., $\alpha=1$ and $\Delta=0$ ).

\section{A. Analysis of $H_{1}$}

Let us start the statistical characterization of the algorithm by considering $H_{1}$. This hypothesis corresponds to assuming $\alpha=1$ and $\Delta=0$, hence (2) reads

$$
\begin{aligned}
x_{i} & =\sum_{m=i M}^{(i+1) M-1} \sqrt{E_{s}} \sum_{k=-\infty}^{+\infty}\left\{\frac{a_{k} c_{m}^{*}}{M} R_{p}\left[(m-k+D) T_{s}\right]\right. \\
& \left.\times e^{\jmath(2 \pi F(m+D)+\varphi)}+\frac{1}{M} n_{m} c_{m}^{*}\right\} \\
& =\frac{\sqrt{E_{s}}}{M} R_{p}\left(D T_{s}\right) \sum_{m=i M}^{(i+1) M-1}\left|c_{m}\right|^{2} e^{\jmath(2 \pi F(m+D)+\varphi)}+\gamma_{i}+\eta_{i} \\
& =\nu_{i}+\gamma_{i}+\eta_{i}
\end{aligned}
$$

where the useful part of the correlation $\nu_{i}$ has been isolated. The two remaining terms are the contribution of the intersymbol interference (ISI) $\gamma_{i}$, defined as

$$
\gamma_{i}=\frac{1}{M} \sum_{m=i M}^{(i+1) M-1} \zeta_{m} c_{m}^{*}
$$

with

$$
\zeta_{m}=\sqrt{E_{s}} e^{\jmath(2 \pi F(m+D)+\varphi)} \sum_{\substack{k=-\infty \\ k \neq m}}^{+\infty} a_{k} R_{p}\left[(m-k+D) T_{s}\right]
$$

and the contribution of the AWGN $\eta_{i}$, defined as

$$
\eta_{i}=\frac{1}{M} \sum_{m=i M}^{(i+1) M-1} n_{m} c_{m}^{*}
$$

The noise component $\eta_{i}$ is complex circularly-symmetric Gaussian-distributed with mean zero and variance

$$
E\left\{\left|\eta_{i}\right|^{2}\right\}=\frac{2 \sigma^{2}}{M}=2 \sigma_{\eta}^{2}
$$

because $\left|c_{m}\right|^{2}=1$ [1]. Therefore, the noise term statistics are independent of the various offsets (phase, timing, and the frequency offsets).

By conditioning with respect to $F$ and $\varphi$, and by invoking the central limit theorem, the random variables $\left\{\zeta_{m}\right\}$ in (6) result to be complex circularly-symmetric Gaussian-distributed with mean zero (because $E\left\{a_{k}\right\}=0$ ) and variance

$$
E\left\{\left|\zeta_{m}\right|^{2} \mid H_{1}, F, \varphi\right\}=E_{s} \sum_{\substack{k=-\infty \\ k \neq 0}}^{+\infty} R_{p}^{2}\left[(k+D) T_{s}\right]=2 \sigma_{\zeta \mid H_{1}}^{2} .
$$

Therefore, the ISI component $\gamma_{i}$ in (5) is a complex circularlysymmetric Gaussian variable with mean zero and variance

$$
E\left\{\left|\gamma_{i}\right|^{2} \mid H_{1}\right\}=\frac{2 \sigma_{\zeta \mid H_{1}}^{2}}{M}=2 \sigma_{\gamma \mid H_{1}}^{2} .
$$

Note that $\left\{\zeta_{m}\right\}$ in (6) are not uncorrelated. However, the terms of $E\left\{\left|\gamma_{i}\right|^{2}\right\}$ having the form $E\left\{\zeta_{m} c_{m}^{*} \zeta_{n}^{*} c_{n}\right\}$ vanish because $E\left\{c_{m}^{*} c_{n}\right\}=0$ for $m \neq n$. It is also worth noting that the ISI variance depends on the fractional timing offset only, which is assumed to be deterministic.

As done before, we first evaluate the statistics of $x_{i}$ in (4) by conditioning with respect to the random frequency offset $F$ and phase $\varphi$. Using the fact that $\left\{\gamma_{i}\right\}$ and $\left\{\eta_{i}\right\}$ are zero-mean, we see that

$$
\begin{aligned}
E\left\{x_{i} \mid H_{1}, F, \varphi\right\} & =\frac{\sqrt{E_{s}}}{M} R_{p}\left(D T_{s}\right) e^{\jmath \varphi} \sum_{m=i M}^{(i+1) M-1} e^{\jmath 2 \pi F(m+D)} \\
& =\sqrt{E_{s}} R_{p}\left(D T_{s}\right) e^{\jmath[2 \pi F(i M+D)+\varphi]} \frac{\operatorname{sinc}(M F)}{\operatorname{sinc}(F)} \\
& =\mu_{x_{i} \mid H_{1}} .
\end{aligned}
$$

The test statistic $\Lambda$ in (3) is then distributed according to the non-central $\chi^{2}$ distribution with $2 L$ degrees of freedom. Each of the component random variables has variance

$$
\begin{aligned}
E\left\{\left|y_{i}\right|^{2} \mid H_{1}, F, \varphi\right\} & =2 \sigma_{y \mid H_{1}}^{2}=\frac{1}{L}\left(2 \sigma_{\gamma \mid H_{1}}^{2}+2 \sigma_{\eta}^{2}\right) \\
& =\frac{1}{L_{P}}\left[E_{s} \sum_{\substack{k=-\infty \\
k \neq 0}}^{+\infty} R_{p}^{2}\left[(k+D) T_{s}\right]+2 \sigma^{2}\right] .
\end{aligned}
$$

Under these assumptions, the probability of correct synchronization (also known as probability of detection), can be expressed in closed form [8] as

$$
\begin{aligned}
P_{D \mid F, \varphi} & =P\left\{\Lambda>\xi \mid H_{1}, F, \varphi\right\} \\
& =Q_{L}\left(\frac{d}{\sigma_{y \mid H_{1}}}, \frac{\sqrt{\xi}}{\sigma_{y \mid H_{1}}}\right)
\end{aligned}
$$


where $Q_{n}(a, b)$ is the Marcum- $Q$ function of order $n$ [9], $\xi$ is a threshold, and $d$ is the non-centrality parameter of the $\chi^{2}$ distribution defined as

$$
\begin{aligned}
d^{2} & =\sum_{i=0}^{L-1}\left|E\left\{y_{i} \mid H_{1}, F, \varphi\right\}\right|^{2} \\
& =E_{s} R_{p}^{2}\left(D T_{s}\right) \frac{\operatorname{sinc}^{2}(M F)}{\operatorname{sinc}^{2}(F)} .
\end{aligned}
$$

The unconditional probability of detection can be computed by numerical integration as

$$
P_{D}=\frac{1}{2 \beta} \int_{-\beta}^{-\beta} Q_{L}\left(\left|R_{p}\left(D T_{s}\right) \frac{\operatorname{sinc}(M F)}{\operatorname{sinc}(F)}\right| \frac{\sqrt{E_{s}}}{\sigma_{y \mid H_{1}}}, \frac{\sqrt{\xi}}{\sigma_{y \mid H_{1}}}\right) \mathrm{d} F
$$

since it is independent of $\varphi$.

A closed-form approximation of (7) can be obtained by a Taylor expansion of the Marcum- $Q$ function. To this purpose, we recall the definition of the Marcum- $Q$ function

$$
Q_{n}(a, b)=\int_{b}^{+\infty} e^{-\frac{z^{2}+a^{2}}{2}}\left(\frac{z}{a}\right)^{n-1} z I_{n-1}(a z) \mathrm{d} z
$$

where $I_{\nu}(x)$ is the modified Bessel function of the first kind of order $\nu$, we define for the sake of notation

$$
\begin{aligned}
k & =\left|R_{p}\left(D T_{s}\right)\right| \frac{\sqrt{E_{s}}}{\sigma_{y \mid H_{1}}} \\
b & =\frac{\sqrt{\xi}}{\sigma_{y \mid H_{1}}} \\
g(F) & =k\left|\frac{\operatorname{sinc}(M F)}{\operatorname{sinc}(F)}\right|
\end{aligned}
$$

and replace (8) in (7). We can rewrite (7) as

$$
\begin{aligned}
P_{D}= & \frac{1}{2 \beta} \int_{-\beta}^{-\beta} \int_{b}^{+\infty} e^{-\frac{z^{2}+g(F)^{2}}{2}}\left(\frac{z}{g(F)}\right)^{L-1} z I_{L-1}(z g(F)) \mathrm{d} z \mathrm{~d} F \\
= & \frac{1}{2 \beta} \int_{b}^{+\infty} e^{-\frac{z^{2}}{2}} z^{L} \int_{-\beta}^{-\beta} e^{-\frac{g(F)^{2}}{2}}(g(F))^{1-L} I_{L-1}(z g(F)) \mathrm{d} F \mathrm{~d} z \\
= & \frac{1}{2 \beta} \int_{b}^{+\infty} e^{-\frac{z^{2}}{2}} z^{L}\left(\frac{z}{2}\right)^{L-1} \\
& -\beta \int_{-\beta} e^{-\frac{g(F)^{2}}{2}}{ }_{0} \tilde{F}_{1}\left(; L ; \frac{z^{2} g^{2}(F)}{4}\right) \mathrm{d} F \mathrm{~d} z
\end{aligned}
$$

where ${ }_{0} \tilde{F}_{1}(; a ; z)$ is the regularized confluent hypergeometric function [10]. Then, we approximate the inner integrand with its fourth-order Taylor expansion evaluated in $F=0$. The solution of the inner integral can be expressed as a linear combination of Marcum- $Q$ and Nuttall- $Q$ functions [11]. The latter is a generalization of the Marcum- $Q$ and is defined as

$$
Q_{m, n}(a, b)=\int_{b}^{+\infty} e^{-\frac{z^{2}+a^{2}}{2}} z^{m} I_{n}(a z) \mathrm{d} z
$$

By resorting to the recursive relation of the Nuttall- $Q$ function [11]

$$
\begin{aligned}
Q_{m, n}(a, b) & =a Q_{m-1, n+1}(a, b)+(m+n-1) Q_{m-2, n}(a, b) \\
& +b^{m-1} e^{-\frac{b^{2}+a^{2}}{2}} I_{n}(a b)
\end{aligned}
$$

and to the relation between the Nuttall- $Q$ and the Marcum- $Q$ functions [11]

$$
Q_{n+1, n}(a, b)=a^{n} Q_{n+1}(a, b)
$$

we can approximate (7) as

$$
\begin{aligned}
P_{D} & \cong A Q_{L}(k, \hat{b})+B Q_{L+1}(k, \hat{b}) \\
& +C \hat{b}^{L+1} e^{-\frac{\hat{b}^{2}+k^{2}}{2}} I_{L-1}(k \hat{b})
\end{aligned}
$$

where $A, B$, and $C$ are normalization constants (omitted for lack of space) depending on $M, L, k$, and $\beta$, and

$$
\hat{b}=\frac{\sqrt{\xi+\varepsilon}}{\sigma_{y \mid H_{1}}} \text {. }
$$

The introduction of the heuristic threshold shift $\varepsilon$ helps in compensating for the error caused by the truncation of the Taylor expansion of the inner integrand in (9). Future investigations will be focused on finding an analytical expression for $\varepsilon$.

\section{B. Analysis of $H_{0}$}

Under the $H_{0}$ hypothesis, the integer timing offset is nonzero, i.e., $\Delta \neq 0$. The sampled signal in (1) becomes then

$$
r_{m}=\alpha \zeta_{m}+n_{m}
$$

where $\zeta_{m}$ is defined in a slightly different way with respect to (6), namely

$$
\zeta_{m}=\sqrt{E_{s}} e^{\jmath(2 \pi F(m+D+\Delta)+\varphi)} \sum_{k=-\infty}^{+\infty} a_{k} R_{p}\left[(k+D) T_{s}\right] .
$$

The output of the correlator $x_{i}$ now reads

$$
\begin{aligned}
x_{i} & =\frac{1}{M} \sum_{m=i M}^{(i+1) M-1} \alpha \zeta_{m} c_{m}^{*}+n_{m} c_{m}^{*} \\
& =\alpha \gamma_{i}+\eta_{i}
\end{aligned}
$$

while the definitions of $\eta_{i}, \gamma_{i}, y_{i}$, and $\Lambda$ remain unchanged. The noise term $\eta_{i}$ has the same statistics computed before, whereas $\zeta_{m}$ is still complex Gaussian-distributed with mean zero but now it has variance equal to

$$
\begin{aligned}
\operatorname{var}\left\{\zeta_{m} \mid H_{0}, F, \varphi\right\} & =2 \sigma_{\zeta \mid H_{0}}^{2} \\
& =E_{s} \sum_{k=-\infty}^{+\infty} R_{p}^{2}\left[(k+D) T_{s}\right]
\end{aligned}
$$


As before, the ISI term $\gamma_{i}$ is complex Gaussian-distributed with mean zero and variance

$$
\operatorname{var}\left\{\gamma_{i} \mid H_{0}\right\}=\frac{2 \sigma_{\zeta \mid H_{0}}^{2}}{M}=2 \sigma_{\gamma \mid H_{0}}^{2} .
$$

By conditioning with respect to $F$ and $\varphi$, the correlator output is therefore a complex Gaussian variable with mean zero and variance

$$
\begin{aligned}
\operatorname{var}\left\{x_{i} \mid H_{0}, F, \varphi\right\} & =2 \sigma_{x \mid H_{0}}^{2}=E\left\{|\alpha|^{2}\right\} 2 \sigma_{\gamma \mid H_{0}}^{2}+2 \sigma_{\eta}^{2} \\
& =\frac{1}{M}\left[p_{s} E_{s} \sum_{k=-\infty}^{+\infty} R_{p}^{2}\left[(k+D) T_{s}\right]+2 \sigma^{2}\right] .
\end{aligned}
$$

As a consequence, the test statistic $\Lambda$ follows the central $\chi^{2}$ distribution with $2 L$ degrees of freedom, each of which having variance equal to $\sigma_{y \mid H_{0}}^{2}=\sigma_{x \mid H_{0}}^{2} / L$. Hence, the probability of false alarm can be computed as

$$
\begin{aligned}
P_{F A} & =P\left\{\Lambda>\xi \mid H_{0}, F, \varphi\right\} \\
& =\exp \left\{-\frac{\xi}{2 \sigma_{y \mid H_{0}}^{2}}\right\} \sum_{k=0}^{L-1} \frac{1}{k !}\left(\frac{\xi}{2 \sigma_{y \mid H_{0}}^{2}}\right)^{k}
\end{aligned}
$$

which is independent of both $F$ and $\varphi$ [8].

\section{Optimization of the correlation length}

For a given probability of false alarm, we will find the couple $\left(\xi_{\text {opt }}, L_{\text {opt }}\right)$ that maximizes the probability of detection. Since $L_{P}=M L$, to each value of $L$ corresponds one single value for $M$. As starting point for the optimization, we will use the value of $M$ provided by the coherent integration length dimensioning (CHILD) rule

$$
M_{\text {child }} \cong \frac{3}{8 F}
$$

which provides an upper bound for the value of $M_{\mathrm{opt}}$ [7]. Since the correlation length provided by the CHILD rule depends on the frequency offset (which has to be known), we adopt as starting point for the optimization the value corresponding to the worst case scenario (i.e., when $F=\beta$ ).

\section{NumericAl Results}

For the sake of simplicity, we assess the performance of the NCPDI on the AWGN channel by assuming absence of fractional timing errors (i.e., $D=0$ ). Since the fractional timing error is assumed deterministic, considering a non-zero value would change the results only quantitatively. This assumption allows us to discard the ISI contribution $\gamma_{i}$ and to consider the thermal noise only as source of nuisance. As operative regime, we focus on the VL-SNR region, which corresponds to the most challenging scenario for the synchronization chain. Namely, we investigate the algorithm performance at SNR equal to $-5 \mathrm{~dB}$. By employing a quadricorrelator as first stage of the chain, a maximum residual normalized frequency offset $\beta$ equal to $5 \%$ has been considered. Even though the quadricorrelator can provide even lower residual frequency offsets, we have chosen such a value so as to make a conservative assumption.

First, we validate the approximation in (10) by comparing it with the exact expression of the average probability of detection in (7), evaluated via numerical integration. As reported in Fig. 2, the approximation is very good even at very low probabilities. The approximation in (10) will be used later in the characterization of the performance of the algorithm.

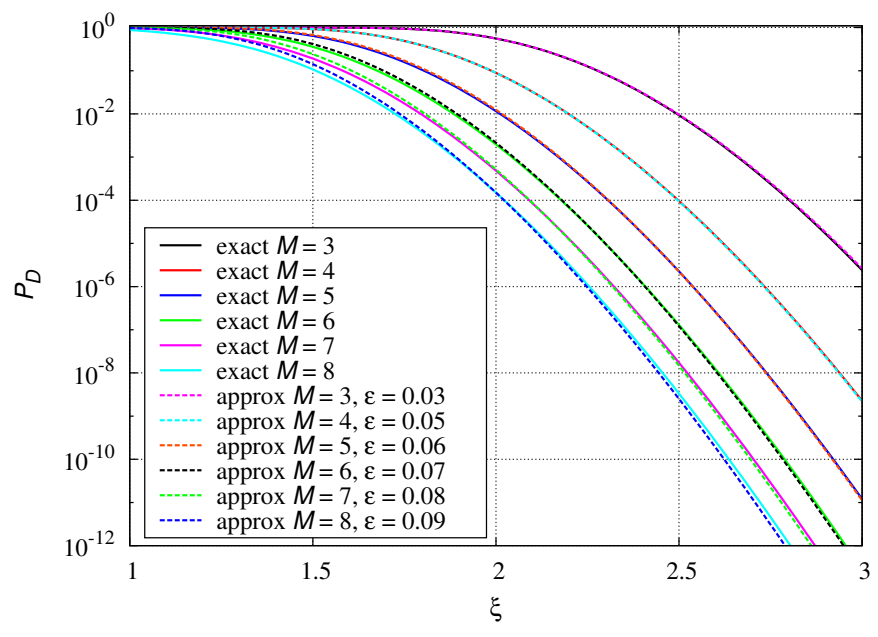

Fig. 2. Exact and approximated probabilities of detection for different values of the correlation length $M$.

Further, we optimize the correlation length $M$ in order to minimize the average probability of missed detection $P_{M D}=$ $1-P_{D}$ for a given probability of false alarm $P_{F A}$. By assuming $P_{F A}=10^{-5}$, we numerically evaluate the threshold $\xi$ and the number of sub-blocks $L$ used in (11). Then, by assuming $L_{P}=270$ (which is the length of the SoSF field in the DVB-S2X standard [1]), we compute the correlation length $M=\left\lfloor L_{P} / L\right\rfloor$, where $\lfloor z\rfloor$ denotes the highest integer lower than $z$. For different values of $M$ we get different thresholds, and we use these to compute the corresponding values of $P_{D}$ by resorting to (10). The resulting curve in Fig. 3 shows that the optimal value for $M$ (at SNR equal to $-5 \mathrm{~dB}$ ) is $M_{\text {opt }}=5$, which is lower than the value obtained by the CHILD rule $M_{\text {child }}=7.5$ in the worst case scenario (i.e., with $F=0.05)$. This result is in apparent contradiction to the CHILD rule in (12), since to a lower (on average) frequency offset should have corresponded a larger value of $M$. However, the CHILD rule does not take into account the signal processing performed by the NCPDI algorithm, and provides only an upper bound to the optimal correlation length $M_{\text {opt }}$ [7]. Indeed, as $M$ increases, $L$ decreases and a sufficient averaging effect in (3) does not occur, leading to higher noise and poorer performance. On the other hand, decreasing $M$ reduces the reliability of the correlation (2), leading to a performance degradation. Moreover, since the curve is steep, a wrong choice of $M$ can have a significant impact on the probability of missed detection.

Finally, the performance of the NCPDI has been assessed 


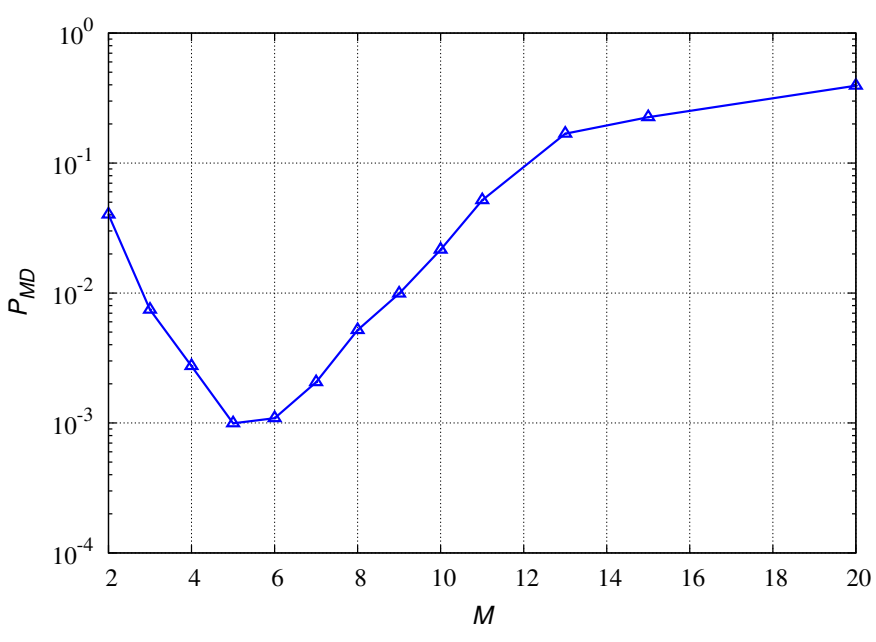

Fig. 3. Probability of missed detection as a function of the correlation length $M$ at SNR $=-5 \mathrm{~dB}$ corresponding to a probability of false alarm equal to $10^{-5}$, for $F$ random uniformly distributed in $[-0.05,0.05]$.

in terms of receiver operating characteristics (ROC), which is the specific performance measure for a detection scheme. For each value of $P_{F A}$, the corresponding values of threshold $\xi$ and correlation length $M$ have been computed by means of (11). As before, $P_{M D}$ has been evaluated by means of (10) and validated via numerical simulations for different values of $\xi$ and $M$. Concerning the numerical simulations, for each point at least 100 missed detections have occurred over a maximum value of simulated frame equal to $10^{7}$. The resulting ROCs are shown in Fig. 4, highlighting the dependence of the optimal correlation length $M$ on $P_{F A}$. Therefore, different systems, having different specifics (e.g., complexity constraints limiting the correlation length $M$ or the number of sub-blocks $L$ ) and/or performance requirements (e.g., different required probabilities of false alarm or missed detection), will have different optimal design parameters.

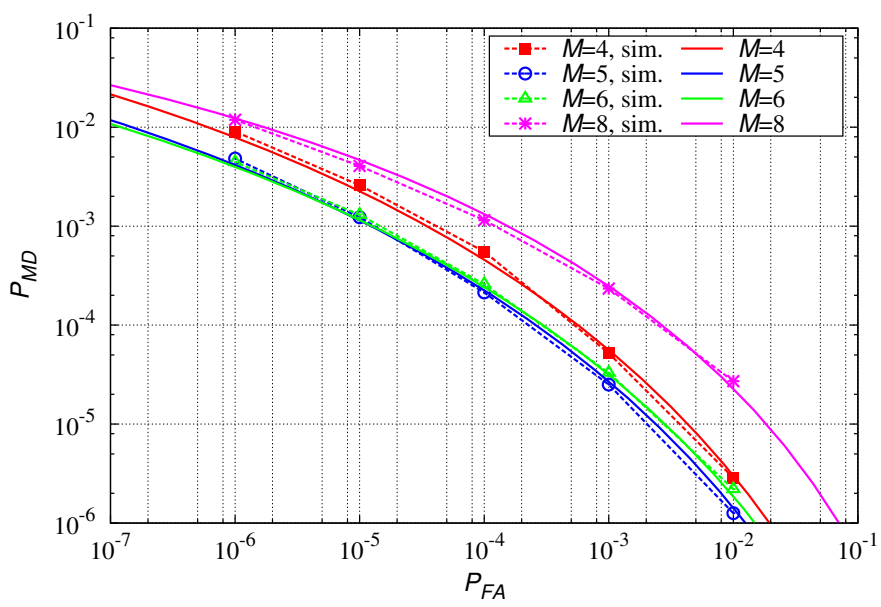

Fig. 4. ROCs for NCPDI at SNR $=-5 \mathrm{~dB}$ and random $F$ uniformly distributed in $[-0.05,0.05]$ for different values of $M$.

\section{Conclusions}

In this paper, we have considered the problem of optimizing the NCDPI algorithm for frame synchronization in presence of an unknown frequency offset. Taking a Bayesian approach, we have modelled the residual frequency offset (i.e., the output of a coarse frequency offset estimator) as a uniformly distributed random variable. In such a scenario, we have derived the exact average probability of detection (in integral form) and a tight closed-form approximation. The derived expression is then used to optimize the NCDPI parameters, and the performance of the resulting detector is enumerated. It can be verified that the proposed methodology provides for a robust design that can be used in a number of challenging scenarios like aeronautical and maritime communications.

\section{ACKNOWLEDGEMENT}

This work was partly funded by the European Space Agency's ARTES-5.1 project "Novel ground components prototype beyond DVB-S2 for broadband satellite networks" (AO/1-7621/13/NL/NR, Activity Reference 6-.016) under the contract $4000110120 / 14 / \mathrm{NL} / \mathrm{NR}$, and by the Erasmus+ Traineeship Program.

\section{REFERENCES}

[1] ETSI EN 302 307-2 Digital Video Broadcasting (DVB), Second generation framing structure, channel coding and modulation systems for Broadcasting, Interactive Services, News Gathering and other broadband satellite applications, Part II: S2-Extensions (DVB-S2X), Available on ETSI web site (http://www.etsi.org).

[2] A. N. D'Andrea and U. Mengali, "Design of quadricorrelators for automatic frequency control systems," IEEE Trans. Commun., vol. 41, no. 6, pp. 988-997, June 1993.

[3] R. Pedone, M. Villanti, A. Vanelli-Coralli, G. Corazza, and P. Mathiopoulos, "Frame synchronization in frequency uncertainty," IEEE Trans. Commun., vol. 58, no. 4, pp. 1235-1246, April 2010.

[4] A. J. Viterbi, CDMA: Principles of Spread Spectrum Communication. Addison-Wesley, 1995.

[5] M. Villanti, P. Salmi, and G. Corazza, "Differential post detection integration techniques for robust code acquisition," IEEE Trans. Commun., vol. 55, no. 11, pp. 2172-2184, Nov 2007.

[6] G. Corazza and R. Pedone, "Generalized and average likelihood ratio testing for post detection integration," IEEE Trans. Commun., vol. 55, no. 11, pp. 2159-2171, Nov 2007.

[7] G. E. Corazza, R. Pedone, and M. Villanti, "Frame acquisition for continuous and discontinuous transmission in the forward link of satellite systems," International Journal of Satellite Communications and Networking, vol. 24, no. 2, pp. 185-201, 2006.

[8] J. Proakis and M. Salehi, Digital Communications, 5th ed., McGrawHill, Ed., 2008.

[9] J. Marcum, "Table Q-functions, U.S. air force project RAND res. memo, m-339, ASTIA document AD 1165451," Rand Corp., Santa Monica, CA, USA, Tech. Rep., 1950.

[10] M. Abramowitz and I. A. Stegun, Handbook of Mathematical Functions with Formulas, Graphs and Mathematical Tables. U.S. Department of Commerce, National Bureau of Standard, Applied Mathematics Series, 1972.

[11] A. Nuttall, "Some integral involving the Q-function," Naval Underwater Systems Center, New London, Connecticut, USA, Tech. Rep., April 1972. 